\title{
Estimation of Relative Pollutant Loads in a Lake Using Neural Network
}

\author{
Ken Hiramatsu ${ }^{1}$, Takayuki Tanaka ${ }^{2}$, Toshihiko Kawachi ${ }^{3}$ and Hiroshi Itagaki ${ }^{4}$
}

\begin{abstract}
Pollution from diffuse sources in lakes and reservoirs has become a serious problem. The location of the diffuse source is still difficult to identify and the load from it is also difficult to estimate because of its inherent properties. The mechanism of the pollution from the diffuse sources is conceptually recognized, but the details, e.g., pathway to lakes and detention period in soil, are still obscure. Among the informative assets available, most of which are uncertain, observed water qualities and climatic conditions at some stations in lakes are certainly well quantified and reliable. This study aims to develop a method to estimate the relative pollutant loads of the diffuse sources using an artificial neural network, in which the observed water qualities and climate are used as keys to the solution. In addition, supplementary tutoring procedure to aid the training of the neural network is introduced to reduce the error in the estimation. The method developed is applied to Lake Biwa under some hypothetical conditions. The results show that the method successfully estimates the relative pollutant loads and hence its significant contribution to help water quality management of lakes will be expected.
\end{abstract}

Keywords: Relative pollutant load; Artificial neural network; Inverse problem; Diffuse source

\section{Introduction}

Since the basic law for environmental pollution control has been established in Japan, serious toxic pollution, e.g., heavy metal spillage, has decreased remarkably in number. In contrast, eutrophication due to nutrients from diffuse sources has become a serious problem, as the standard of living has been improved. For example, serious proliferation of water bloom called 'aoko' has still occurred every spring and summer in Lake Biwa located in Shiga prefecture, though prefectural government has prohibited the use of phosphorus abluent since 1979. It clearly indicates that nutrients are flowing into the lake not only from residential area but also from widely spread diffuse sources, i.e., forests, farmland and so on.

Generally speaking, identification of the diffuse source and estimation of its pollutant load are more difficult than those of point source, because the amount of pollutant per unit area is fairly small and the area of source may be considerably large. In addition, the pathway from the source to the lake is also obscure.

As a matter of fact, there are some researches on identification of the point source(s) in surface water and groundwater [3],[4],[5], but those for the diffuse source(s) are very few.

Under these circumstances, only water qualities and climatic conditions, observed at some stations in the lake, are more obvious and reliable. Therefore,

\footnotetext{
${ }^{1}$ Associate Professor, Faculty of Agriculture, Gifu University, 1-1 Yanagido, Gifu, 501-1193 Japan

${ }^{2}$ Engineer, Ministry of Agriculture, Forestry, and Fisheries of Japan, 1-2-1 Kasumigazeki, Chiyoda-ku, Tokyo, 100-8950 Japan

${ }^{3}$ Professor, Graduate School of Agricultural Science, Kyoto University, Kitashirakawa-oiwake-cho, Sakyo-ku, Kyoto, 6068502 Japan

${ }^{4}$ Professor, Faculty of Agriculture, Gifu University, 1-1 Yanagido, Gifu, 501-1193 Japan
}

development of the method to identify the location of the diffuse sources and to estimate the load from them with reference to the observed water qualities and climatic conditions is an urgent need. It could be a tool to prevent the lake from eutrophicating.

In this paper, a method to find relative pollutant loads using an artificial neural network (ANN) in which observed water quality and climate are considered as inputs is presented, and applied to Lake Biwa under some hypothetical conditions.

\section{Methodology}

\subsection{Basic concept}

Pollutant that is a certain kind of nutrients or ions is assumed to be discharged into a lake from surrounding diffuse sources, simultaneously. The method is developed that can estimate relative pollutant load of each source from the concentration of pollutant and climatic conditions observed at some stations in the lake. The relative pollutant load is defined herein as a ratio of pollutant that comes out from each source to the total. ANN is introduced as a main processor of the method in which each relative pollutant load and the concentration of pollutant will be output and input, respectively.

The method takes three steps, i.e., training, calibration and estimation. In the training process, inputs and corresponding outputs are prepared by flow and pollutant- transport analyses and then the ANN learns about the non-linear relationship between them. In the calibration, the trained ANN is applied to the trial couples of input and output that are different from the data used for training. Trained ANN is expected to successfully estimate the relative load from the observed pollution. However, re-training or supplementary training is introduced when the resultant outputs and observed ones do not 
match well. Finally, in the estimation, pollution observed at the stations are cast into the well-trained ANN to estimate the relative pollutant loads.

The process of estimating relative pollutant loads is illustrated in Figure 1.

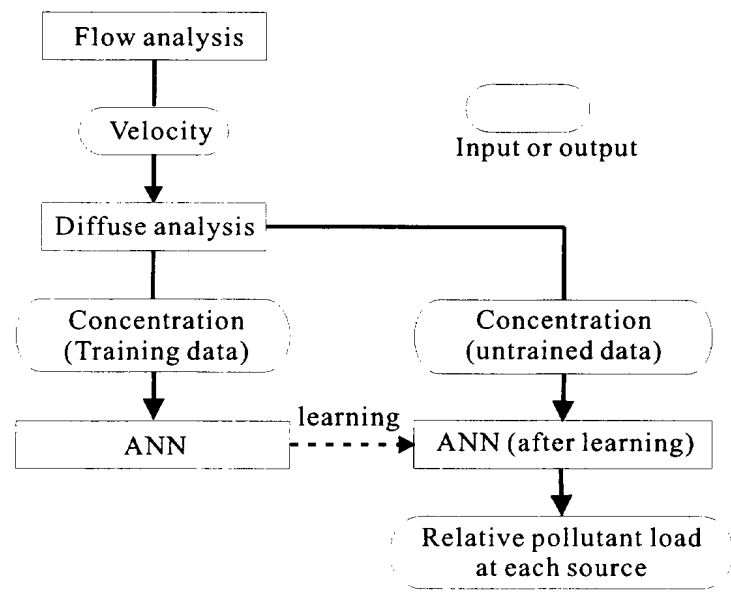

Figure 1: Diagram of the method

\subsection{Artificial neural networks [1]}

Artificial neural networks (ANN) is a kind of artificial intelligence technology that has information processing characteristics similar to the biological neuron. ANN is configured from a number of parallel processors called neuron. The neurons are linked to each other with respective strengths that are determined by training as in Figure 2. The data used in the training consist of input data and output data whose relations are a priori known.

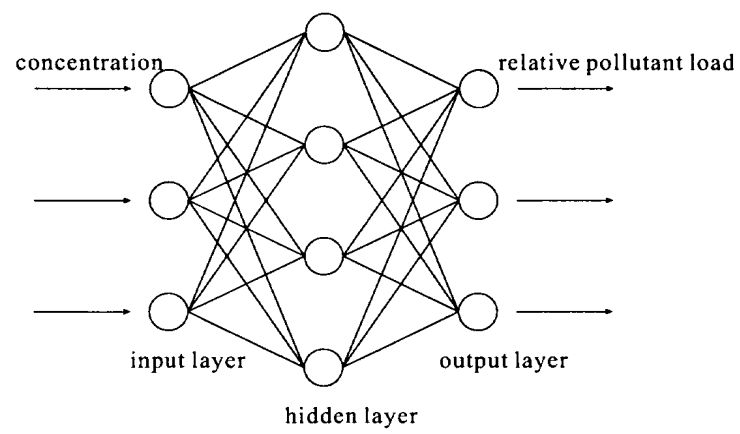

Figure 2: Hierarchy ANN and back propagation

The training continues until the error $E$ given by (1) becomes small enough.

$$
E=\frac{1}{2} \sum_{j}\left(O_{j}-t_{j}\right)^{2}
$$

where $O_{j}$ is computed output and $t_{j}$ is the desired data at neuron $j$ in output layer. $O_{j}$ is expressed as

$$
\begin{gathered}
O_{j}=f\left(y_{j}\right) \\
f(y)=\frac{1}{1+e^{-\alpha y}}
\end{gathered}
$$

$$
y_{j}=\sum_{j=1}^{n} w_{i j} x_{i}-\theta
$$

where $w_{i j}$ is weight between neurons, $x_{i}$ is output of hidden layer, $f(y)$ is activation function (sigmoidal function), $\theta$ is a threshold value of sigmoidal function and $\alpha$ is a coefficient of sigmoidal function.

\subsection{Multiple-level model [2]}

At least training data have to be prepared by the aid of numerical models, because actual pollution cannot be brought about for the experiments. In this study, the multiple-level model, which can consider the vertical velocity or diffusion, is employed in order to reproduce the movement of pollutant as realistic as possible. Namely, the level system is introduced in the vertical direction as shown in Figure 3 and the horizontal movements of water and pollutant are integrated vertically within each level.

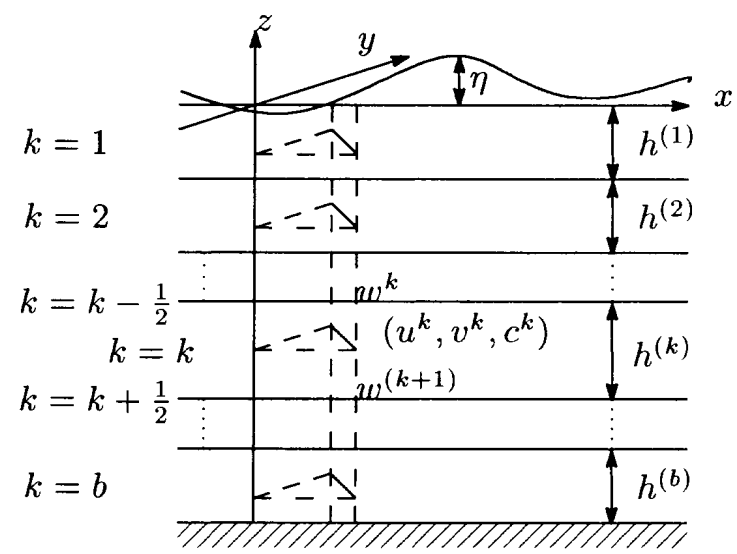

Figure 3: Level system

The equations of momentum, continuity and pollutant transport are given as (5) to (12) for each level.

$$
\begin{gathered}
\frac{\partial u_{i}^{(k)}}{\partial t}+u_{j}^{(k)} \frac{\partial u_{i}^{(k)}}{\partial x_{j}}+\frac{1}{h^{(k)}}\left(\left(u_{i} w\right)^{(k-1 / 2)}\right. \\
\left.-\left(u_{i} w\right)^{(k+1 / 2)}\right)+\frac{1}{\rho} \frac{\partial p^{(k)}}{\partial x_{i}}-f \epsilon_{i j} u_{j}^{(k)} \\
=\frac{\partial \tau_{i j}^{(k)}}{\partial x_{j}}+\frac{1}{\rho h^{(k)}}\left(\tau_{i}^{(k) u}-\tau_{i}^{(k) l}\right) \\
p^{(k)}=\rho g \eta-\rho g\left(h^{(k-1)}+\frac{1}{2} h^{(k)}\right) \\
\left(u_{i} w\right)^{(k-1 / 2)}=w^{(k)} \frac{1}{2} \cdot\left(u_{i}^{(k)}+u_{i}^{(k-1)}\right) \\
\tau_{i j}^{(k)}=K^{(k)}\left(\frac{\partial u_{i}^{(k)}}{\partial x_{j}}+\frac{\partial u_{j}^{(k)}}{\partial x_{i}}\right) \\
\tau_{i}^{(k+1 / 2)}=f_{m} \rho\left(u_{i}^{(k)}-u_{i}^{(k+1)}\right)\left|u_{i}^{(k)}-u_{i}^{(k+1)}\right|
\end{gathered}
$$




$$
\begin{gathered}
\frac{\partial \eta}{\partial t}+\sum_{l=1}^{b} \frac{\partial\left(h^{(l)} u_{i}^{(l)}\right)}{\partial x_{i}}=0 \\
w^{(k)}=-\sum_{l=k}^{b} \frac{\partial\left(h^{(l)} u_{i}^{(l)}\right)}{\partial x_{i}}
\end{gathered}
$$

where $x_{i}(i=1,2)$ is $x, y$ coordinate, $t$ is time, $u_{i}^{(k)}$ is the vertically averaged horizontal velocity in $(k)$ th level, $w^{(k)}$ is the velocity component for $z$ direction at boundary between $(k)$ th level and $(k+1)$ th level, $h^{(k)}(k=1, \cdots, b)$ is the thickness of $(k)$ th level, $\eta$ is the water surface elevation measured from the mean water elevation, $b$ is the number of level, $\rho$ is the density of water, $p^{(k)}$ is pressure in the $(k)$ th level, $g$ is the gravitational acceleration, $K^{(k)}$ is the turbulent viscous coefficient, $f$ is the Coriolis parameter, $\epsilon_{i j}$ is Edington's epsilon function, $\tau_{i}^{(k+1 / 2)}$ is turbulent shear stress at the boundary between $(k)$ th level and $(k+1)$ th level and $f_{m}$ is the friction coefficient.

$$
\begin{aligned}
\frac{\partial c^{(k)}}{\partial t} & +u^{(k)} \frac{\partial c^{(k)}}{\partial x}+v^{(k)} \frac{\partial c^{(k)}}{\partial y} \\
& +\frac{1}{h^{(k)}}\left((w c)^{(k-1 / 2)}-(w c)^{(k+1 / 2)}\right) \\
& =\frac{\partial}{\partial x}\left(D_{x} \frac{\partial c^{(k)}}{\partial x}\right)+\frac{\partial}{\partial y}\left(D_{y} \frac{\partial c^{(k)}}{\partial y}\right) \\
& +\frac{1}{h^{(k)}} D_{z}\left(\left(\frac{\partial c}{\partial z}\right)^{(k-1 / 2)}-\left(\frac{\partial c}{\partial z}\right)^{(k+1 / 2)}\right)
\end{aligned}
$$

where $c$ is pollutant concentration in the $(k)$ th level and $D_{x}, D_{y}, D_{z}$ are diffusion coefficients in the respective directions.

In regard to the boundary condition, the Non-slip boundary is employed between the water area and the land area and the friction of the bottom is given as follow

$$
\tau_{i}^{(k+1 / 2)}=\tau_{i}^{(b)}=f_{b} \rho u_{i}^{(b)}\left|u_{i}^{(b)}\right|
$$

where $f_{b}$ is the friction coefficient of the bottom. And at the surface level, the wind friction is introduced as

$$
\tau_{i}^{(1 / 2)}=c \rho_{a}\left|W_{i}\right| W_{i}
$$

where $c$ is wind drag coefficient, $\rho_{a}$ is density of air and $W_{i}$ is wind velocity.

\section{Application}

\subsection{Target domain}

The method for estimation of relative pollutant loads is applied to Lake Biwa[6] under some hypothetical conditions. Though a number of rivers feed Lake Biwa, the following five rivers, i.e., Yasu, Ane, Ado, Hino and Echi, having the largest catchment areas in the basin, are considered as representative inlets for the lake as shown in Figure 4. River Seta is a unique outlet. Total discharge to Lake Biwa is $\mathbf{1 5 6 . 5}$ $\left(\mathrm{m}^{3} / \mathrm{s}\right)$ and it is shared out among the five rivers in proportion to their catchment area as in Table 1 . It is assumed that pollutants over the diffuse sources gather to their nearest inlet and drain into to the lake, though the diffuse sources are scattered over their catchment area. Therefore, the five rivers substitute for the diffuse sources in this application of the method.

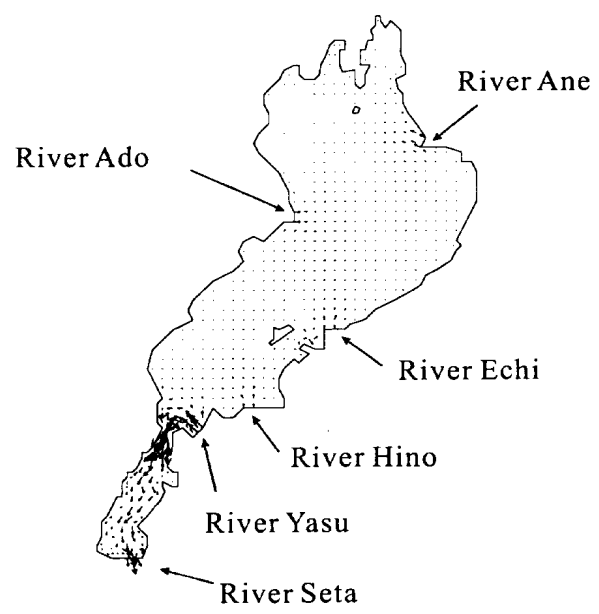

Figure 4: Location of rivers and currents velocities at the surface level

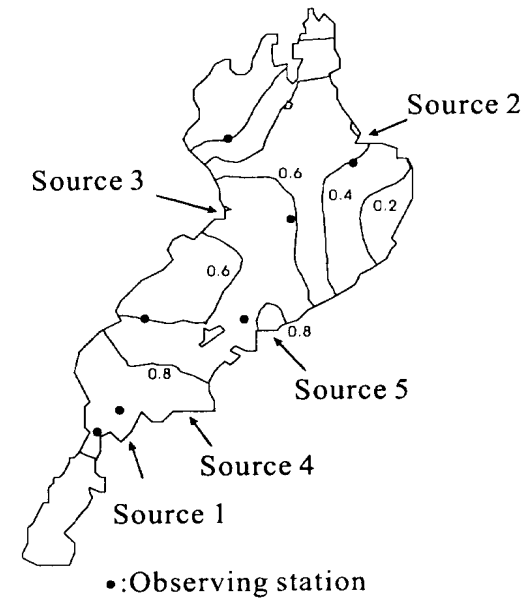

Figure 5: Initial pollutant concentrations and observing stations

Table 1: Catchment area and river discharge

\begin{tabular}{|c|c|c|}
\hline River & Catchment area $\left(\mathrm{km}^{2}\right)$ & Discharge $\left(\mathrm{m}^{3} / \mathrm{s}\right)$ \\
\hline Yasu & 382.2 & 41.0 \\
\hline Ane & 369.0 & 39.6 \\
\hline Ado & 306.9 & 33.0 \\
\hline Hino & 210.9 & 22.7 \\
\hline Echi & 202.3 & 20.2 \\
\hline
\end{tabular}




\subsection{Flow and advective diffusion analyses}

At river mouths, the water surface elevation measured from the mean water elevation $\eta$ is assumed to be $0.0(\mathrm{~m})$. In the flow analysis, five wind-blowing patterns (wind velocity $=0.0(\mathrm{~m} / \mathrm{s}), 6.0(\mathrm{~m} / \mathrm{s})$ from the north, the south, the east and the west) are considered. The currents computed for the five patterns are cast into the pollutant-transport analysis to generate the training and calibration data. In generating the data, four options are considered, viz., 1) which source loads an abnormal amount of pollutant, 2) how long the flux of pollutant is loaded, 3) how much the flux is loaded and 4) to which direction wind drives the pollutant. Figure 4 shows the current velocity distribution at the surface level of wind velocity $w=0.0(\mathrm{~m} / \mathrm{s})$.

Table 2: Parameters for flow analysis

\begin{tabular}{|l|c|c|c|}
\hline parameter & symbol & value & unit \\
\hline water density & $\rho$ & $1.0 \times 10^{3}$ & $\left(\mathrm{~kg} / \mathrm{m}^{3}\right)$ \\
\hline atmospheric density & $\rho_{a}$ & 1.29 & $\left(\mathrm{~kg} / \mathrm{m}^{3}\right)$ \\
\hline Coriolis parameter & $f$ & $1.0 \times 10^{-4}$ & $(\mathrm{rad} / \mathrm{s})$ \\
\hline internal friction coeff. & $f_{m}$ & $1.0 \times 10^{-3}$ & $(-)$ \\
\hline bottom friction coeff. & $f_{b}$ & $2.3 \times 10^{-3}$ & $(-)$ \\
\hline wind drag coeff. & $c$ & $1.0 \times 10^{-3}$ & $(-)$ \\
\hline gravitational accel. & $g$ & 9.8 & $\left(\mathrm{~m} / \mathrm{s}^{2}\right)$ \\
\hline time step & $\Delta t$ & 20 & $(\mathrm{~s})$ \\
\hline
\end{tabular}

The initial distribution of pollutant concentration is required before generating the training and calibration data. In this study, the condition given by 50 days computation with the flux of 0.01 (dimensionless) at each source and wind of $w=0.0 \mathrm{~m} / \mathrm{s}$ is employed as the initial condition. Figure 5 shows the initial distribution of pollutant concentration and the location of observing stations.

\subsection{Configuration of ANN}

Inputs for neural network are rates of concentrationchange at 7 observing stations and 2 horizontal components of wind velocity. The rate of concentrationchange is defined as concentration of pollutant at 7 th day divided by the initial concentration at each station. Therefore, the number of neurons in the input layer becomes 9 and that in the output layer is 5 identical to the number of pollutant loading sources. The desired data for training of neuron $j\left(t_{j}\right)$ is the relative pollutant load at each source, which is defined as

$$
t_{j}=\frac{m_{j}}{m_{1}+m_{2}+m_{3}+m_{4}+m_{5}}
$$

where $m_{j}$ is the total amount of pollutant discharging from a source $j$ during last 7 days.

\subsection{Training and calibration}

ANN is trained with 450 patterns of autoptic data and calibrated with 250 pattern of unlearned data.
The overall relative square error, $e^{2}$, between estimated and true values of the relative load, evaluated by (16), turns out to be 0.457 .

$$
e^{2}=\sum_{j=1}^{5} e_{j}^{2}=\sum_{j=1}^{5}\left\{\left(a_{j}-b_{j}\right) / b_{j}\right\}^{2}
$$

where $e_{j}$ is relative error between estimated and true values for source $j, a_{j}$ is estimated value and $b_{j}$ is true value.

Figure 6 shows a sample bar graph of estimated and true values. Through all the computated results, the relative pollutant loads at the highly polluting sources tend to be over-estimated. Figure 7 shows the relation between $a_{j}$ and $e_{j}$, which is linearly approximated by

$$
e_{j}=0.580 a_{j}-0.116
$$

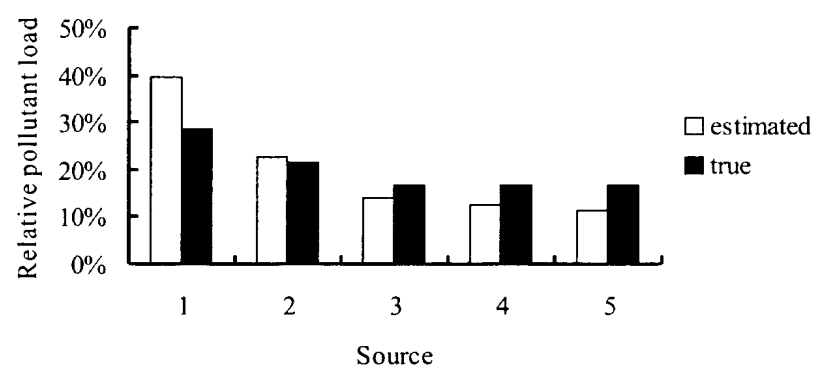

Figure 6: Estimated and true values of relative load

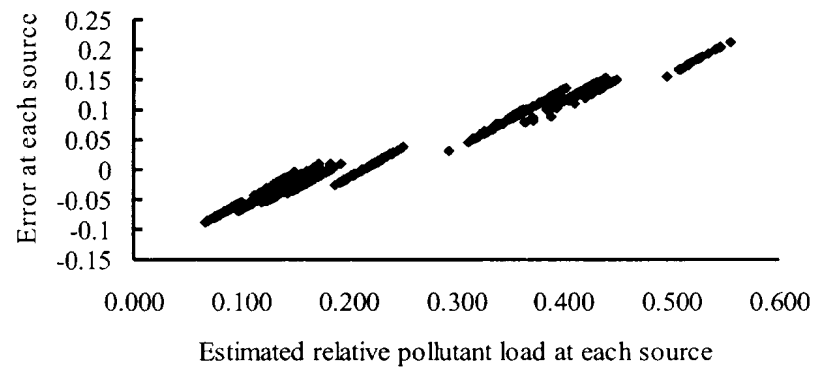

Figure 7: Relation between errors and estimated values

Due to this fact, estimated values are corrected using (17).

In casting the autoptic data into the ANN, some of the physical and geographical information drop out from the process of the estimation, which may be a cause of the error. Therefore, the correction can be considered as a supplementary tutoring procedure for ANN.

\subsection{Estimation}

Under ordinary circumstances, the estimation should be executed for actual pollution occuring in the lake. However, due to the lack of real data, 50 unlearned trial data are used which are generated by numerical 
methods and are used in neither training nor calibration procedure.

The overall relative error becomes 0.003 with the supplementary tutoring procedure, while it turns out to be 0.7926 without the procedure. A sample of computed results is illustrated in Figure 8 to show this difference.

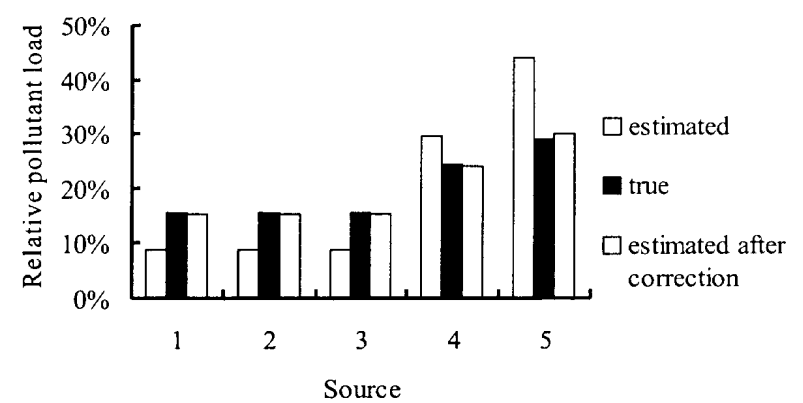

Figure 8: Estimated values with/without correction and true values

\section{Conclusions}

As known well, ANNs offer a powerful means of solving poorly defined problems in which the mechanism between input and output is not clear or lost. The method whose core procedure is ANN has successfully been implemented to the problem of estimating relative pollutant loads from diffuse sources. The results computed with the aid of supplementary tutoring procedure conform to the true values quite exactly. It is, however, hardly adequate, because the unknown trial data have been generated by the same numerical methods as the training data and therefore they have almost the same deflective tendency. On the other hand, the actual observed data may have similar tendency as long as the numerical models represent the phenomenon properly. In any case it is of great use to overcome the difficulty in learning by introducing supplementary procedures. Application with the real data is of considerable significance to complete the method and ensure the reliability of it.

As is often the case with ANN, the physical mechanism that certainly exists is neglected in its process and only both ends, viz., input and output are emphasized. However, known information, e.g., location of rivers, types of land-use in watershed area, etc., should be included into the method explicitly and positively. It will be a clue to improve the developed method that just provides the first step to manage the diffuse pollution at present.

\section{References}

[1] ASCE (1994): Special Issue; Neural Networks, J. Comp. in Civil Eng., Vol.8, No.2, pp.129-265.

[2] Kodama, T. and Kawahara, M. (1992): Multiple Level Finite Element Anaysis for Tidal Current Flow with Non-Reflective Open Boundary Conditions, Proc. JSCE, 446, pp.89-99.

[3] Hiramatsu, K., Kawachi, T., Kurimoto, H., Fazal, M. A. and Fukatsu, M. (1996): Numerical Estimation of Water Polluting Points Using Neural Networks, Hydrodynamics, edited by Chwang et al., Balkema, Rotterdam, pp.1069-1074.

[4] Kurimoto, H. (1997): Identification of Pollutant Loading Source Using Neural Networks, Master's Thesis, Kyoto University.

[5] Mahar, P. S. and Datta, B. (2001): Optimal Monitoring Network and Ground-Water-Pollution Source Identification, J. of Water Resources Planning and Management, ASCE, Vol.127, No.1, p.20-29.

[6] Somiya, I. (2000): Environment and Water Quality Formation in Lake Biwa, Gihodo Press. (in Japanese).

[Discussion open until June 30, 2003] 